\title{
Can a structured checklist prevent problems with laparoscopic equipment?
}

\author{
E. G. G. Verdaasdonk $\cdot$ L. P. S. Stassen · \\ W. F. Hoffmann - M. van der Elst • \\ J. Dankelman
}

Received: 4 November 2007/Accepted: 26 May 2008/Published online: 3 July 2008

(C) The Author(s) 2008

\begin{abstract}
Background A high incidence of problems with the technical equipment is known to occur during routine laparoscopic procedures. Use of a structured checklist of preparatory measures could help to prevent these problems. This study aimed to determine the extent to which a checklist reduced the number of incidents with technical laparoscopic equipment.

Methods A 28-item checklist was developed based on frequently occurring laparoscopic equipment problems during 30 laparoscopic cholecystectomies (the control group). A further 30 procedures were conducted with the checklist (the checklist group). The number and type of incidents with the technical equipment were compared between the groups. All the procedures were recorded using a special audio-video system (black-box).

Results In the checklist group, the total number of incidents per procedure was 53\% lower than in the control group (23/30 versus 49/30). The checklist led to fewer incidents of wrong positioning (9/30 versus 22/30), and wrong settings and connections (7/30 versus $12 / 30)$ of the equipment. Defects or malfunctions decreased from 15/30 in the control group to $7 / 30$ in the checklist group. One or more incidents with the equipment occurred in $47 \%$ (14/ 30 ) of the checklist procedures compared with $87 \%(26 / 30)$
\end{abstract}

E. G. G. Verdaasdonk ( $\bowtie)$ · L. P. S. Stassen · J. Dankelman Department of BioMechanical Engineering,

Faculty of Mechanical, Maritime \& Materials Engineering, Delft University of Technology, Delft, The Netherlands

e-mail: e.g.g.verdaasdonk@tudelft.nl

E. G. G. Verdaasdonk · L. P. S. Stassen .

W. F. Hoffmann - M. van der Elst

Department of Surgery, Reinier de Graaf Group, Delft,

The Netherlands of the control procedures. Median time taken to complete the checklist items was $3.3 \mathrm{~min}$ (range 1.0-8.3 min).

Conclusions Use of a checklist was feasible and helped to reduce problems with the laparoscopic equipment in the operating room. Future research should aim to implement checklists for different procedures and investigate their effects.

The introduction of minimally invasive surgery (MIS) and its sophisticated technical equipment have made the surgical environment more complex. New problems have been created in the domain of man-machine interaction during these high-technology procedures, creating opportunities for errors or incidents to occur. Surgeons are aware of the existence of user problems with the laparoscopic equipment and instruments in their operating theatres [1]. Recently, the occurrence of incidents with the technical equipment has been quantified [2]. These incidents consisted of problems with the mechanical instruments and problems with the technical equipment, for example positioning, absence or malfunctioning. The frequency of incidents was strikingly high: 49 incidents in 26 out of 30 routine laparoscopic cholecystectomies.

A short preoperative checklist based on the concepts used in industry and aviation could help to prevent these problems. Currently, no checklists that specifically aim to structure human interaction with the surgical equipment in the operating room are available. It is unknown whether this approach is feasible and effective.

The aim of this study was to develop a structured preoperative checklist and to determine the feasibility of its use and whether it could help to prevent incidents with laparoscopic equipment during routine laparoscopic procedures. 


\section{Methods}

Development of the checklist

A concept checklist was developed based on an incident analysis with the equipment in 30 laparoscopic cholecystectomies (the control group) [2]. Guidelines for aviation checklist design were also taken into consideration [2, 3]. The concept checklist was reviewed and adjusted by an expert laparoscopic surgeon and several experienced operating room nurses. In a pilot study with two procedures the concept checklist was tested, adjusted on the basis of errors and inconveniences. The size was decreased from A4 to A5 format and all pages were covered with transparent plastic cover.

Table 1 and Fig. 1 show the 28 -item checklist that was considered ready for further investigation. The checklist consisted of three parts and was to be executed by the circulating operating room nurse. Part 1 consisted of the checks necessary prior to the arrival of the patient at the operating room. Part 2 covered the period up to the point when the endoscopic camera was introduced into the abdomen. Part 3 was to be executed after the camera had been introduced into the abdomen.

\section{Study protocol}

The study was conducted in the setting of a large non-university training hospital. Our standard laparoscopic equipment consisted of a laparoscopic tower trolley and two Sony PVM-Trinitron color video monitors. The tower trolley held an insufflator, a xenon light source, a digital three-chip camera, and a camera unit. Diathermy equipment was mounted separately on another trolley. Each team consisted of a surgical trainee, a (supervising) surgeon, a scrub nurse, and a circulating nurse. The checklist was used during 30 laparoscopic cholecystectomies (the checklist group). All procedures were recorded using a special audio-video system that consisted of two digital video recorders with three camera image inputs and two microphones (Storz, Tuttlingen, Germany). Patients were asked to sign an informed consent form on the day prior to the surgical procedure.

The video material was reviewed and analyzed by the first author (E.G.G.). Procedures that needed to be converted to open or conventional cholecystectomies were analyzed up to the moment of removal of the trocars.

Assessment method

Incidents with the technical equipment, such as the insufflator, the diathermy equipment, monitors, light source, endoscope or suction unit were divided into several categories: position and absence (equipment in the wrong position or not present at all), settings and connection (problems due to wrong settings or connections), and defects and unclear (problems due to a defects or unclear malfunctioning). Problems with the laparoscopic instruments were not included. Time taken to execute the items on the checklist was also measured.

The number of incidents in the checklist group was compared to the number in the control group. The Fisher exact test (two-tailed) was used to analyze differences in total number of incidents between the checklist and the control group. $p<0.05$ was considered to be statistically significant.

\section{Results}

Between June 2004 and December 2004, 30 laparoscopic cholecystectomies without the use of a checklist were recorded and analyzed [2]. These procedures formed the control group. From 1 September 2006 to 30 March 2007 a total of 30 laparoscopic cholecystectomies with the use of checklist were recorded and formed the checklist group. In the control group, the surgeons were 7 different staff surgeons and 11 surgical trainees. In the checklist group, the surgeons were 6 different staff surgeons and 12 surgical trainees.

In four procedures in the control group, the laparoscopic approach was converted to an open procedure [2]. These decisions were not due to technical problems with the equipment or instruments. None of the procedures in the checklist group were converted to an open procedure.

In the checklist group, the total number of incidents per procedure was $53 \%$ lower than that in the control group (checklist group 23/30 versus control group 49/30). Figure 2 displays the number of incidents in the checklist group and control group. In the checklist group there were fewer incidents of wrong positioning of the equipment (9/ 30 versus $22 / 30$ ) and wrong settings or connections (7/30 versus 12/30). The number of incidents due to defects or unclear malfunctioning was $7 / 30$ in the checklist group versus $15 / 30$ in the control group. Table 2 shows the number of incidents in more detail in relation to the different components of the laparoscopic equipment.

Overall, one or more incidents occurred with the equipment in $47 \%(14 / 30)$ of the procedures in the checklist group compared with $87 \%(26 / 30)$ in the control group ( $p=0.003$ ). Furthermore, twice as many incidents occurred in the first 10 procedures of the checklist group than in the last 20 procedures (Fig. 3). None of the problems observed on the recordings caused direct operative complications in the patients.

Median time taken to complete the items on the checklist was $3.3 \mathrm{~min}$ (range $1.0-8.3 \mathrm{~min}$ ). 
Table 1 Structured checklist of preparatory measures

\section{Part I: Preparation prior to the procedure}

$\square$ Check presence of image on two monitors

No image then:

$\rightarrow$ Switch monitors off and on

$\rightarrow$ Connect cable (BNC) from "video comp" to first monitor

"input: comp/sos" on back of second monitor

No image then:

$\rightarrow$ Switch monitors off and on

$\rightarrow$ Replace laparoscopic tower without image and report problem

$\square$ Check the amount of $\mathrm{CO}_{2}$ gas available (min. 1 green line is sufficient for one standard laparoscopic procedure)

Make sure that:

$\rightarrow$ The TEM cable is not connected

$\rightarrow$ Gas valve is open (turned to the left)

$\square$ Check whether $\mathrm{CO}_{2}$ filter is present on insufflator

$\square$ Check whether light source is functioning

Light source defective then:

$\rightarrow$ Replace laparoscopic tower and report problem

\section{Part II: Before introduction of 1st trocar}

Equipment set-up

$\square$ Place diathermy equipment at foot on right-hand side of the patient

$\square$ Place footswitch near right foot of surgeon, cable directed along upper side of pillar of the OR table

$\square$ Place first monitor and laparoscopic equipment tower near right shoulder of the patient

$\square$ Check with surgeon whether monitor is correctly positioned.

$\square$ Place second monitor on the left shoulder of the patient

$\square$ Check with assisting surgeon whether monitor is correctly positioned.

$\square$ Place suction equipment next to diathermy equipment.

Equipment connections \& settings

Monitors

$\square$ Check again the presence of an image on the monitors

No image then:

$\rightarrow$ Switch monitors off and on

$\rightarrow$ Connect cable (BNC) from "video comp" to first monitor "input: comp/ sos" No image then:

$\rightarrow$ Switch monitors off and on

$\rightarrow$ Replace laparoscopic tower without image and report problem

Insufflator \& light source

$\square$ Connect light cable to light source

$\square$ Set light source at $75 \%$, check again the presence monitor image

$\rightarrow$ If necessary adjust to $100 \%$

$\square$ Check that disposable $\mathrm{CO}_{2}$ with filter is connected to insufflator cable

$\square$ Set maximal insufflator pressure to $14 \mathrm{mmHg}$

$\square$ Set minimal insufflation flow to $1.4 \mathrm{l} / \mathrm{min}$
Equipment connections \& settings

Diathermy ValleyLab Force FX: monopolar

$\square$ Connect diathermy cable of laparoscopic instrument to coagulation at front port 2

$\square$ Connect foot switch cable to the back in port 2

$\square$ Connect disposable patient-plate

$\square$ Connect diathermy knife (yellow) to "cut/coa" at the front

$\square$ Set minimal "coagulation" on 35 Watt (lower setting allowed)

$\square$ Set minimal "cut" to 35 Watt

Note: if diathermy device malfunctions during the procedure

$\rightarrow$ First check above items

$\rightarrow$ Then replace diathermy cable of laparoscopic instrument

$\rightarrow$ Then replace foot switch + cable and report problem

$\rightarrow$ Replace diathermy equipment and report problem

\section{Part III: After introduction of 1st trocar}

$\square$ On indication of surgeon that trocar is correctly placed in the abdomen, set flow at maximal

$\square$ Perform "white balance"

$\square$ Focus endoscopic camera

$\square$ Cross-check with surgeons whether light and color settings are correct

If color setting incorrect despite "white balance"

$\rightarrow$ Switch monitor off and on and consult the operation team

Color settings still incorrect then:

$\rightarrow$ Adjust settings on monitor

Image to dark:

$\rightarrow$ Light source higher

$\rightarrow$ Replace light cable. Set source at $75 \%$

$\rightarrow$ Replace endoscope

$\rightarrow$ Replace camera

$\rightarrow$ Postpone procedure, replace laparoscopic tower and report problem

$\square$ Coat endoscope with anti-condensation fluid

\section{Discussion}

This is the first study on the effects of a short preoperative structured checklist that specifically aimed to prevent problems with the laparoscopic equipment.
The concept of using a structured checklist prior to a surgical procedure is not completely new in health care. Anaesthesia checklists to prevent machinery problems appeared more than a decade ago. Studies showed that various checklist concepts detected machinery faults in 50- 
Fig. 1 Checklist concept

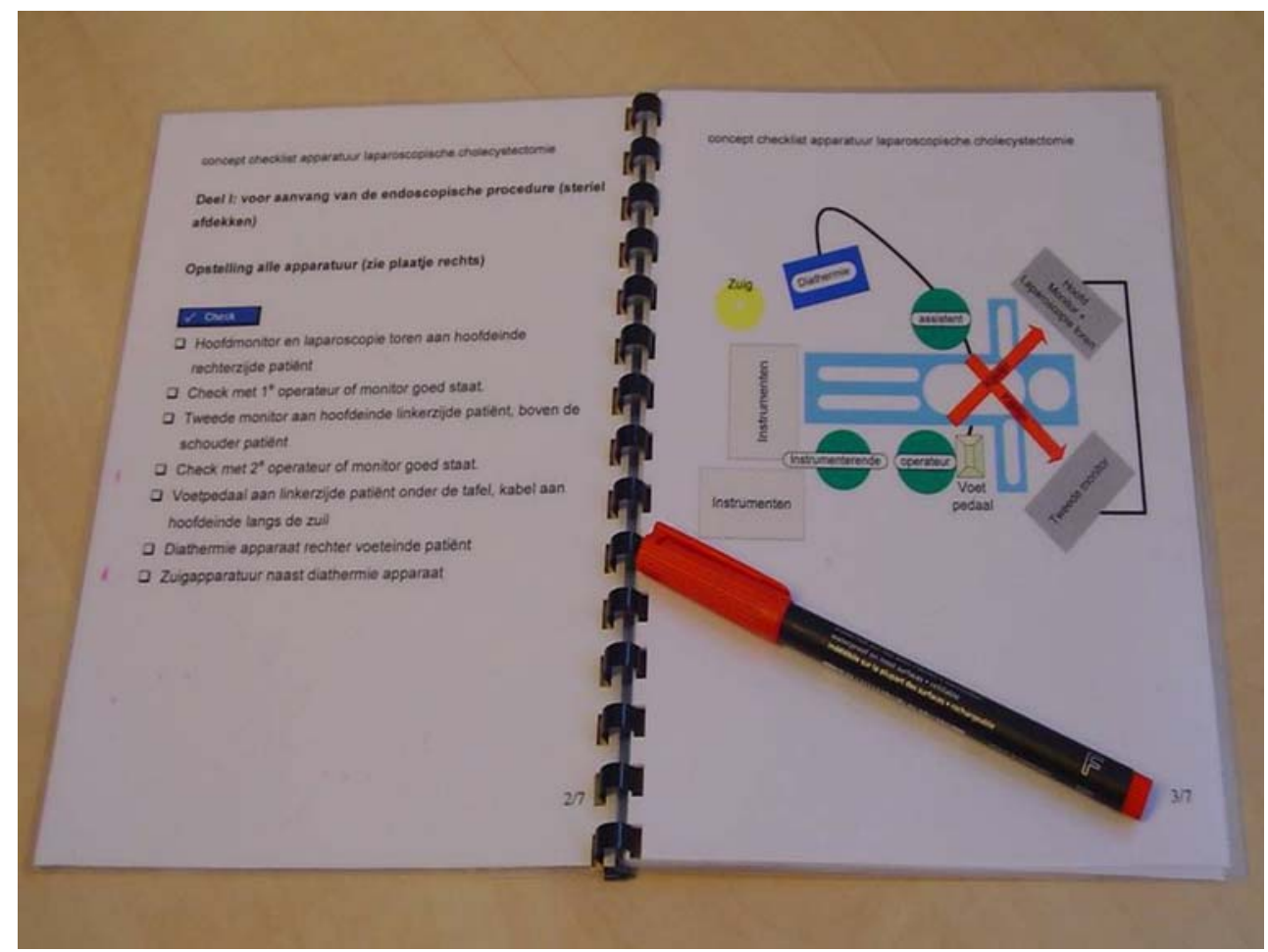

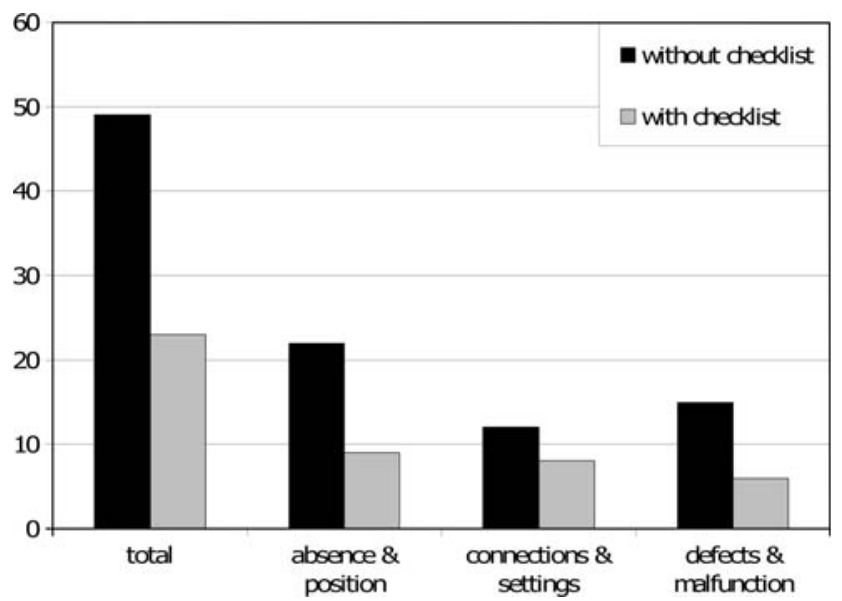

Fig. 2 Number of incidents with and without the use of a checklist
$80 \%$ [4-6]. The lists or protocols varied between hospitals vary and it was not clear whether some hospitals ever used these lists.

In 2004, the Joint Commission on Accreditation of Health care Organizations (JCAHO) mandated the Universal Protocol to prevent wrong site, wrong procedure, and wrong person surgery within all JCAHO-accredited organizations [7]. The protocol consists of guidelines that advise a preoperative verification process, marking of the operative site, and time-out before starting the procedure [8]. The use of a checklist in the time-out period is especially recommended. The results of the first studies that evaluated the effect of structured checklists were positive and indicated improved safety climate, decreased wrong site surgery, and improved personnel satisfaction and team

Table 2 Number of incidents with the equipment in the control group and checklist group

\begin{tabular}{|c|c|c|c|c|c|c|c|c|}
\hline & \multicolumn{2}{|l|}{ Subtotal } & \multicolumn{2}{|c|}{ Absence \& position } & \multicolumn{2}{|c|}{ Settings \& connections } & \multicolumn{2}{|c|}{ Defects \& unclear } \\
\hline & Control & Checklist & Control & Checklist & Control & Checklist & Control & Checklist \\
\hline Monitor/Image & 24 & 14 & 13 & 5 & 3 & 3 & 8 & 6 \\
\hline Endoscope & 1 & 0 & & & & & 1 & \\
\hline Light source & 1 & 0 & & & & & 1 & \\
\hline Insufflator & 4 & 4 & & & 4 & 3 & & 1 \\
\hline Diathermy & 10 & 1 & & & 5 & 1 & 5 & \\
\hline Pedals & 9 & 4 & 9 & 4 & & & & \\
\hline Total & 49 & 23 & 22 & 9 & 12 & 7 & 15 & 7 \\
\hline
\end{tabular}




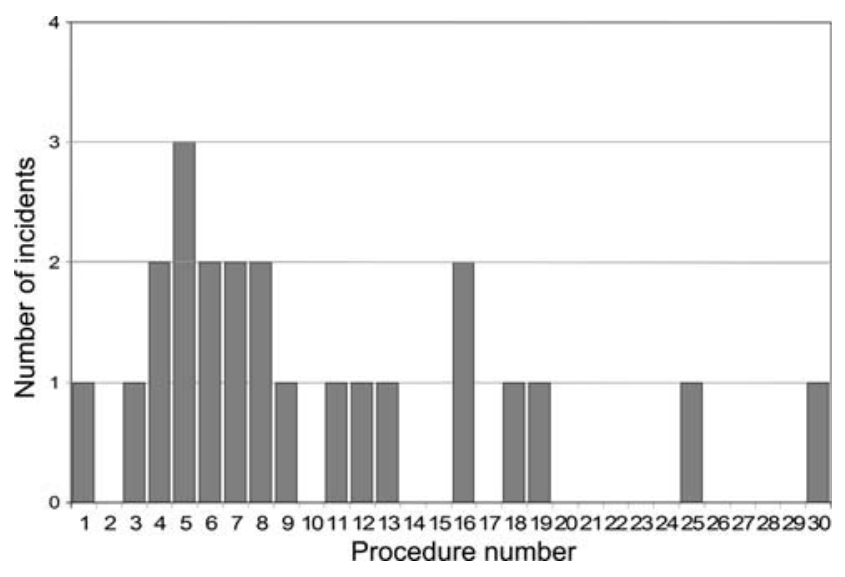

Fig. 3 Number of incidents per procedure in the Checklist group

cohesion [9-11]. However, there were no specific guidelines for the physical design of the checklist.

Although the advantages of checklists seem logical, critics doubt their benefit and still emphasize the disadvantages, such as the extra time needed to complete the list, the extra work, and the rigidity of following a specific list. Nevertheless, when safety is the highest priority, the potential benefits should outweigh these disadvantages.

The checklist used in this study led to a more than 50\% reduction in the number of incidents with the equipment. In a retrospective report by Kwaan [12], two-thirds of the wrong-site surgeries ( 8 out of 13) could have been prevented by the correct use of the JCAHO protocol. In the case of wrong-side surgery, it is difficult to determine the effect of the Universal Protocol solely on the basis of the decrease in adverse events, because wrong-side surgery is extremely rare (1 in 112,994 operations). As was shown in the present study, the checklist could not prevent all incidents with the laparoscopic equipment. In the literature, the studies on checklist for anaesthesia equipment also showed that they could not detect or eliminate all the faults. Therefore, the use of checklist does not guarantee complete safety. However, in this study more than half the incidents were prevented, while with the anaesthesia equipment, even up to $80 \%$ were prevented, which cannot be interpreted in any other way than a substantial improvement in the quality of care. Furthermore, the effect of a checklist goes beyond any directly measurable reduction in actual adverse events. In the hectic environment of an operating room (OR), with the constraints and separate priorities of each profession, a standardized checklist provides a structure for communication and performance. Moreover, a checklist enhances consciousness about safety issues and awareness of the importance of preventing human errors. The actual incorporation of checklists in daily practice, however, requires the commitment of all personnel. In general, health care professionals are not used to performing tasks and communicating in a standardized manner. Successful incorporation of a preoperative checklist or time-out, therefore, requires cultural changes. In our study, this learning-curve effect was illustrated, with twice as many incidents in the first 10 procedures as in the last 20 procedures. Personnel needed time to become accustomed to the checklist and realize its usefulness.

Another important factor in successful incorporation of a checklist in the OR, is the cooperation of the surgeon [9, 10]. It is considered essential that the checklist initiative is supported by the surgeon. In the study by Markary several "champion physicians" were appointed to ensure the execution of the checklist. This strategy seemed to be effective and it was advised to assign enthusiastic leaders to initiate the protocol.

In the present study, video monitoring was used to observe the level of commitment to the checklist. In addition, a researcher was present during each procedure. This could have slightly influenced the results, because the supervision itself may have enhanced the commitment to the checklist use. However, this influence was present in the control group as well and therefore cannot explain the observed differences.

A relatively high incidence rate occurred in the category "absence and positioning of monitor and pedals" (Table 2). This high rate is mainly caused by wrong positioning of the equipment. Before the introduction of the checklist, the surgical team routinely positioned the monitor and pedal after the procedure was started. Another reason for the high incidence rate was the fact that the wire connecting the diathermy and the pedal was relatively short. This prevented positing the diathermy apparatus close to the patient, which conflicted with the process of sterile draping of the patient. In order to resolve this, the wire was lengthened.

Some time elapsed between the groups being compared in this study. This is because the problems with the equipment came to our attention only after we recorded the first 30 procedures. Structured analysis of the video material was time consuming. In addition, we thoroughly investigated the literature on checklist design before we conducted this study. It is not a straightforward process to develop an effective checklist.

Once the positive effect of protocols or checklists has been acknowledged, it is important to address the question of how to ensure that the checklist is used and that its execution is correct. In aviation, correct checklist use is extensively practised during preflight training. Furthermore, during the flight the cockpit communication is recorded by the voice recorder and stored in the "black box". The video monitoring used in the present study can be considered as a black box and a control mechanism to 
stimulate adherence. Currently, such video or audio monitoring is not standard in most operating rooms, but this may change in the near future once the legal obstacles have been resolved and the skepticism among specialists has ceased. Further research should focus on the design of reliable and effective interfaces that aim to achieve maximal compliance of the users to the protocols. Future studies should also investigate in detail how many incidents are being detected by the use of a checklist and corrected prior to the procedure. In addition, other initiatives can be expected to enhance the cultural changes needed to improve patient safety and outcome such as (OR) team training, critical communication exercises, and education on how human factors influence performance (crew resource management). Currently, we are developing checklists for all types of laparoscopic procedures that are performed in our institution. The checklist for the laparoscopic cholecystectomy in this study is being used as the basis for the design.

In conclusion, it was feasible to employ a preoperative checklist to help prevent problems with laparoscopic technical equipment in the operating room and there was a considerable reduction in the number of incidents. Future research should aim to find the preferred physical presentation and interfaces for such protocols and to implement checklists for different procedures.

Open Access This article is distributed under the terms of the Creative Commons Attribution Noncommercial License which permits any noncommercial use, distribution, and reproduction in any medium, provided the original author(s) and source are credited.

\section{References}

1. Den Boer KT, de Jong T, Dankelman J, Gouma DJ (2001) Problems with laparoscopic instruments: opinions of experts. J Laparoendosc Adv Surg Tech A 11(3):149-55

2. Verdaasdonk EG, Stassen LP, van der Elst M, Karsten TM, Dankelman J (2007) Problems with technical equipment during laparoscopic surgery. An observational study. Surg Endosc 21(2):275-279

3. Cival Aviation Authority (CAA) (2006) Guidance on the design presentation and use of emergency and abnormal checklists. CAP 676. Safety Regulation Group. http://www.caa.c0.uk. Accessed Sept 2007

4. Barthram C, McClymont W (1992) The use of a checklist for anaesthetic machines. Anaesthesia 47(12):1066-1069

5. Manley R, Cuddeford JD (1996) An assessment of the effectiveness of the revised FDA checklist. Aana J 64(3):277-282

6. Kendell J, Barthram C (1998) Revised checklist for anaesthetic machines. Anaesthesia 53(9):887-890

7. Saufl NM (2004) Universal protocol for preventing wrong site, wrong procedure, wrong person surgery. J Perianesth Nurs 19(5):348-351

8. Torkington J, Smith SG, Rees BI, Darzi A (2000) The role of simulation in surgical training. Ann R Coll Surg Engl 82(2):88-94

9. Lingard L, Espin S, Rubin B, Whyte S, Colmenares M, Baker GR, Doran D, Grober E, Orser B, Bohnen J, Reznick R (2005) Getting teams to talk: development and pilot implementation of a checklist to promote interprofessional communication in the OR. Qual Saf Health Care 14(5):340-346

10. Makary MA, Mukherjee A, Sexton JB, Syin D, Goodrich E, Hartmann E, Rowen L, Behrens DC, Marohn M, Pronovost PJ (2007) Operating room briefings and wrong-site surgery. J Am Coll Surg 204(2):236-243

11. DeFontes J, Surbida S (2004) Preoperative safety briefing project. Permanente J 8(2):21-27

12. Kwaan MR, Studdert DM, Zinner MJ, Gawande AA (2006) Incidence, patterns, and prevention of wrong-site surgery. Arch Surg 141(4):353-357; discussion 357-358 